\title{
Analizando el clima educativo en Uruguay: un estudio exploratorio de la cohorte PISA 2012
}

\author{
School climate in Uruguay. \\ An exploratory analysis of PISA 2012 cohort
}

Análise do clima educacional no Uruguai: uma análise exploratória da coorte PISA 2012

\author{
ISSN 1688-9304 - DOI: https://doi.org/10.18861/cied.2019.10.2.2910 \\ Leonel Rivero*1 \\ https://orcid.org/0000-0002-3199-3901
}

Fecha de recibido: 07/08/2018

Fecha de aprobado: 26/05/2019

\section{Resumen}

La presente investigación analiza el clima educativo de los centros relevados por PISA en la cohorte 2012. El clima educativo es un concepto trabajado en las últimas dos décadas a nivel uruguayo y latinoamericano que vincula la sociología de la educación -a través de su preocupación por la incidencia del centro educativo en los resultados- con las ciencias de la educación abocadas al análisis de la generación de espacios saludables para docentes y estudiantes.

El análisis consta de dos niveles, uno descriptivo y otro correlacional, de los constructos. Dichos constructos son los siguientes: clima educativo (reflejado en sus variables Problemas vinculados a docentes y Problemas vinculados a estudiantes), y dos asociados que son Moral docente y Consenso docente.

Por una parte los hallazgos confirman la prevalencia, en la visión de los directores, de algunos elementos problematizados por la literatura tales como la dificultad de enseñar a estudiantes con niveles heterogéneos, el ausentismo docente o la motivación de los estudiantes. Por otra parte, otorgan menor importancia a elementos tales como el malestar de los docentes o la severidad de los mismos en su trato con los estudiantes, lo que sugiere líneas de investigación a profundizar.

A nivel metodológico, los tres constructos de PISA (Clima, Moral y Consenso Docente) muestran correlaciones positivas entre sí con sentido, lo que confirma desde la empiria los supuestos lógicos que subyacen a la construcción teórica del clima educativo como una gran variable.

Palabras clave: clima educativo, PISA, enseñanza media

\begin{abstract}
This research analyzes the educational climate of the schools surveyed by PISA in the 2012 cohort. The educational climate is a concept worked upon during the last two decades both at Uruguayan and at Latin American levels, and which links the sociology of education -through its concern for the incidence of institutions in the results- to the sciences of education devoted to the analysis of the generation of healthy spaces for teachers and students.

The analysis consists of two levels of the school constructs: a descriptive one and a correlational one. The constructs under study were the Educational climate (reflected in its variables Problems linked to Teachers, and Problems linked to Students), and two associated constructs: Teachers Morale, and Teachers Focus.
\end{abstract}


On one hand, findings confirm the prevalence, in the headmasters' view, of some elements problematized by literature, such as the difficulty of teaching students with heterogeneous levels, teachers absenteeism, or student motivation. On the other hand, they give less importance to elements such as teacher discomfort or their severity in dealing with students, which suggests lines of investigation to be studied in depth.

At a methodological level, the three constructs of PISA (Climate, Morale, and Teachers Focus), show positive correlations between each other, which agrees with data on the logical assumptions that underlie the theoretical construction of the educational climate as a great variable.

Key words: School climate, PISA, Middle education

\section{Resumo}

Essa pesquisa analisa o clima educacional dos centros pesquisados pelo PISA na coorte de 2012. O clima educacional é um conceito trabalhado nas últimas duas décadas no Uruguai e na America Latina, na sociologia da educação, preocupada pelo impacto do centro educacional nos resultados educativos; e nas ciências da educação, focadas na análise de espaços saudáveis para professores e alunos.

A análise consta de dois níveis: um descritivo e um correlacional dos construtos do centro: Clima educacional (refletido em suas variáveis ligadas a professores e problemas ligados a alunos) e dois construtos associados: Ensino moral e Consenso de ensino. Os resultados confirmam a prevalência, na visão dos diretores, de alguns elementos questionados pela literatura, como a dificuldade de ensinar a alunos com níveis heterogêneos, não comparecimento de professores, ou ausência de motivação nos alunos. Aliás, eles se importam menos com elementos como desconforto do professor ou seu rigorismo ao lidar com os alunos, o que sugere linhas de pesquisa a aprofundar. No nível metodológico, os três construtos do PISA (Clima, Moral e Consenso de Ensino), mostra correlações positivas entre si, o que confirma, do ponto de vista empírico, os pressupostos lógicos subjacentes à construção teórica do clima educacional como uma variável com sentido.

Palavras-chave: clima educacional, PISA, Ensino médio

\section{Introducción}

El presente artículo analiza la problemática del clima educativo a la luz de información generada a partir del cuestionario PISA 2012 en el Uruguay. Esto se enmarca en una larga tradición nacional que estudia el clima educativo como variable central para comprender tanto el habitar escolar como los resultados educativos (Fernández, 2004; 1999; 1996).

En la modernidad, el proyecto educativo latinoamericano ha transformado sus objetivos y enfrentado diversas problemáticas (Tedesco, 2000) que cuestionan la legitimidad de la institución escolar (Dubet, 2006). En términos generales, este fenómeno ha sido estudiado desde los enfoques que se explicarán a continuación. En primer lugar, desde los análisis de la violencia en el espacio escolar, interpelando los saberes y prácticas habituales de los docentes (Duschatzky, 2013; Kaplan, 2012; 2009), como fenómenos de convivencia (Viscardi y Alonso, 2015; 2013), o de violencia institucional (Viscardi y Habiaga, 2017; Martinis y Falkin, 2017; Rivero, 2015, 2013; Charlot, 2008).

En segundo lugar, en los estudios sobre el sufrimiento docente. Sea ya entendido como malestar (Esteve, 2006, 1987; Rossi Silva, 2001), aislamiento (Fullan y Hargreaves, 1999) u otros enfoques, esta problemática se expresa en el actual descontento de los docentes con su trabajo (INEEd, 2014).

En tercer lugar, el estudio del clima educativo cobra relevancia con el énfasis de la emocionalidad en el ámbito escolar. Esto tiene un fuerte anclaje en la relevancia de las 
habilidades socioemocionales en el aprendizaje (Farrington et al., 2012; Durlak, Weissberg, Dymnicki, Taylor y Schellinger, 2011; INEEd, 2015) que enfatizan el análisis situado de los procesos de enseñanza-aprendizaje (McDermott, 2001).

Por último, desde la sociología de la educación se destaca la utilidad de estudiar el efecto de la institución en los resultados educativos. Desde una temprana línea de estudio asociada a la reproducción social (Bourdieu, 1995; Coleman, Hoffer y Kilgore, 1982), estudios nacionales han probado el efecto de la institución en los resultados académicos (Ravela et al., 1999), la desafiliación escolar (Filardo y Mancebo, 2013; Fernández, 2010) y la distribución del saber docente (Filgueira et al., 2014).

En tal sentido cobra relevancia el análisis de la institución escolar en sus múltiples dimensiones (Frigerio, Poggi y Tiramonti, 1992). En particular surge con relevancia el concepto de clima educativo como una cuestión central para comprender la dinámica cotidiana de los centros educativos y, a través de ella, los diversos fenómenos que tienen lugar en el espacio escolar.

\section{Antecedentes sobre clima educativo}

Dentro de los diversos enfoques que podrían adoptarse para analizar los factores institucionales del centro educativo se ha optado aquí por la noción de clima educativo. El fundamento refiere a que tal concepto ha generado un corpus teórico adecuado para abordar ordenadamente los múltiples elementos que acontecen en la institución educativa además de contar con veinte años de validación a nivel nacional, lo que permite disponer de un acumulado empírico y metodológico (Fernández, 2004, 1999; Blanco, 2009).

La noción de clima educativo tiene antecedentes en el movimiento denominado escuelas eficaces. En el mismo se intentaba estudiar los procesos escolares y las formas en que estos incidían en el aprendizaje. Como señala Blanco (2009), dentro de los elementos comprendidos en la noción de eficacia escolar se incluyen los sentidos de comunidad, liderazgo educativo, clima escolar y aula, altas expectativas, calidad del currículo, organización del aula, estrategias de enseñanza, aprendizaje organizativo, desarrollo profesional y seguimiento y evaluación, así como el compromiso e involucramiento de la comunidad educativa.

Basado en estos elementos Blanco define clima escolar como aquello que "refiere a la integración de la organización, como un atributo que permite coordinar sus operaciones más allá de la estructura y los mecanismos formales. Esto implica que los miembros comparten significados objetivos, valores, normas y motivos" (Blanco, 2009, p. 682).

Otros autores como Milicic y Arón (2014) entienden que el espacio escolar se asemeja al escenario de una novela en la que los actores interactúan según los guiones que se les presentan. Dado que son determinados por circunstancias superiores a las voluntades individuales, los contextos pueden potenciar el desempeño de su papel o generar efectos contraproducentes. De este modo definen el clima escolar como "la percepción que los individuos tienen de los distintos aspectos del ambiente en el cual desarrollan sus actividades habituales, en este caso, el colegio" (Milicic y Arón, 2016, p. 3).

En este marco, es posible realizar una distinción de acuerdo a las dinámicas generadas en el espacio escolar, que las autoras definen como climas tóxicos y climas nutritivos:

"Los climas nutritivos son aquellos que generan climas en que la convivencia social es más positiva, en que las personas sienten que es agradable participar, en que hay una buena disposición a aprender y a cooperar, en que los estudiantes sienten que sus crisis emocionales pueden ser contenidas, y que en general contribuyen a que aflore la mejor 
parte de las personas [mientras que los climas tóxicos] son aquellos que contaminan el ambiente contagiándolo con características negativas que parecieran aflorar las partes más negativas de las personas. En estos climas, además, se invisibilizan los aspectos positivos y aparecen como inexistentes, y por lo tanto existe una percepción sesgada que amplifica los aspectos negativos, y las interacciones se tornan cada vez más estresantes e interferentes con una resolución de conflictos constructiva" (Milicic y Arón, 2014, p. 4).

En sintonía con esto, autores como Fernández (2004) analizan el clima escolar a partir de cuatro elementos: el tamaño del centro, la diferenciación jerárquica y funcional dentro del mismo, las formas de gestión y los mecanismos de control y sanción. A partir de ellos postula que:

“El clima constituye el trasfondo de sentidos compartidos, pre-comprensiones que respaldan aproblemáticamente los acuerdos y las acciones individuales o colectivas que emprenden los miembros de una organización (maestros, administrativos y alumnos). En el sentido de Habermas, se trata de trasfondos para el entendimiento con vistas a la acción sobre el "mundo objetivo", el "mundo social" y el "mundo interior"; una acción que en términos sistémicos suele ser evaluada en términos de "éxito" (por ejemplo, en los aprendizajes)" (Fernández, 2004, p. 55).

En un importante desarrollo conceptual, el autor concibe el clima escolar a partir de tres esferas (Fernández, 2004):

En primer lugar la cultura, que incluye tanto las formas en que se concibe la disciplina como la necesidad de actualizarse, de dar lugar a la participación o a las expectativas sobre los alumnos. Del mismo modo incluye la visión de la escuela y las expectativas de logro.

La segunda esfera comprende la fuerza de los lazos sociales del grupo, potenciándolos o debilitándolos en aspectos tales como la confianza, el apoyo, la reciprocidad, la creatividad y la cooperación o la solidaridad, tanto entre maestros (endo-grupales) como entre maestros, alumnos y padres (exo-grupales). Esta esfera incluye también la cooperación en el centro, el cuidado de los alumnos y la identificación grupal.

La tercera esfera refiere al sentido de afiliación y refuerzo vocacional en el clima y se compone de tres elementos: el tamaño del centro, la antigüedad del personal, y las horas de equipo socioeducativo (psicólogos, trabajadores sociales y otras figuras) para trabajar en la institución.

Con estos criterios Fernández construye dos tipos ideales: la escuela-comunidad y la escuela anómica. Ambas se diferencian según sus niveles de integración, consenso entre docentes, compromisos para con sus estudiantes en riesgo, aislamiento y motivación.

Estas formas de conceptualizar el clima educativo son de gran utilidad para situar la problemática en un espacio disciplinar entre la sociología y las ciencias de la educación. El clima educativo funciona, entonces, como una variable que permite analizar el funcionamiento macro-educativo pero también como una categoría del sentido común de quienes habitan el espacio escolar. Sobre esta conceptualización general PISA desarrolla su propio corpus teórico, que se consigna a continuación.

\section{Marco teórico de PISA}

PISA utiliza una serie de variables pragmáticas sobre clima educativo. Esto supone no contar con un marco teórico cerrado sino enfocarse en una serie de elementos sobre clima que 
la literatura marca como relevantes. Por este motivo resulta central incorporar visiones teóricas que profundicen en el tema para interpretar los resultados arrojados.

Lo relevado por PISA no presenta grandes diferencias con antecedentes reseñados sino que encuentran diversos puntos de contacto, por lo que los conceptos desarrollados tienen utilidad heurística para la interpretación de los resultados. De hecho, algunos de los autores han generado importantes líneas de trabajo a partir de ellas (Fernández y Ríos, 2014).

La sección E del cuestionario sobre centros realizado a directores incluye diversas preguntas de interés sobre el fenómeno de estudio, las que incluyen temáticas tales como inasistencias de los estudiantes, faltas de respeto a docentes, uso de alcohol y drogas, motivación estudiantil, bajas expectativas docentes, prácticas docentes, vínculos con los padres y la comunidad educativa y valoración docente, entre otras. Para analizar esto se exponen a continuación los principales constructos.

\section{Clima educativo (en sentido estricto)}

Como primer elemento, se toma la parte inicial de dicha sección (P17), compuesta por 19 ítems.

Los primeros 8 forman un índice de problemas de clima relacionados con estudiantes, que son los siguientes:

1) Inasistencia a clase sin autorización;

2) Estudiantes que saltean algunas clases;

3) Estudiantes que llegan tarde a las clases;

4) Estudiantes que no asisten a actividades obligatorias (ej. actividades deportivas) o salidas didácticas;

5) Estudiantes que faltan el respeto a los docentes;

6) Estudiantes que entorpecen el desarrollo de las clases;

7) Uso de alcohol o drogas ilegales por parte de estudiantes;

8) Estudiantes que intimidan o amenazan a otros estudiantes, y

9) Estudiantes que no están siendo motivados para que desarrollen todo su potencial. Los restantes once ítems forman un índice de problemas de clima relacionados con docentes:

10) Relaciones inadecuadas entre estudiantes y profesores;

11) Profesores que tienen que enseñar a estudiantes con distintos niveles de habilidad en una misma clase;

12) Profesores que tienen que enseñar a alumnos de diversos orígenes étnicos (ej. lenguaje, cultura) en una misma clase;

13) Bajas expectativas de los profesores respecto de sus estudiantes;

14) Profesores que no atienden las necesidades individuales de los estudiantes;

15) Ausentismo docente;

16) Resistencia al cambio por parte del plantel del centro educativo;

17) Profesores que son excesivamente severos con los estudiantes;

18) Profesores que llegan tarde a clases, $y$

19) Profesores que no están bien preparados para las clases.

Como es posible apreciar, la mayoría de los ítems tienen una correspondencia cercana con los marcos teóricos reseñados. Asimismo, se incorporan elementos que demuestran correspondencia pragmática con el clima lo que hace que, en conjunto, PISA presente una batería de ítems robusta, con buena comparabilidad entre países (OECD, 2014). 
Además de las variables desarrolladas expresamente en el constructo de clima educativo, el clima de un centro está conformado por otras dos variables que presentamos a continuación.

\section{Moral docente}

La forma en que los docentes se sienten en su espacio de trabajo afecta el desarrollo de su función. Por tanto, se entiende que una alta moral del personal docente es clave para el desarrollo del proyecto institucional y también un elemento constitutivo del clima.

A tales efectos, PISA incluye cuatro ítems en el cuestionario de directores, que deben ser completados con la siguiente escala de valores: muy de acuerdo, de acuerdo, en desacuerdo y muy en desacuerdo.

Dichos ítems son:

a) La moral de los profesores en este centro educativo es alta;

b) Los profesores trabajan con entusiasmo;

c) Los profesores están orgullosos de este centro educativo;

d) Los profesores valoran el desempeño académico.

\section{Consenso docente}

Otro de los elementos importantes reseñados por la literatura refiere al consenso entre los educadores acerca de las prioridades, metas y objetivos educativos que deben alcanzarse en la institución.s

Para mensurar esto PISA generó tres ítems en torno a la temática, evaluados a partir de una escala de cuatro posiciones: muy de acuerdo, de acuerdo, en desacuerdo y muy en desacuerdo. Estos ítems son los siguientes:

a) Los profesores de Matemática están interesados en probar nuevos métodos y prácticas de enseñanza;

b) Existe consenso entre los profesores de Matemática de que es mejor adaptar los estándares académicos a los niveles y necesidades de los estudiantes, y

c) Existe consenso entre los profesores de Matemática en que el desarrollo social y emocional de los estudiantes es tan importante como la adquisición de destrezas matemáticas y conocimiento en las clases de Matemática.

A partir de los elementos reseñados se busca desarrollar dos objetivos. El primero, analizar la distribución de los ítems que conforman cuatro constructos sobre clima educativo realizados por PISA. Se entiende que los mismos brindan información de gran interés para conocer las características del clima educativo en los centros de enseñanza media. El segundo, estudiar si existen correlaciones entre estos constructos, conformando empíricamente un metaconstructo sobre clima escolar. Ello permitiría entender el clima escolar como una variable relevante con sentido propio y no como resultado de fuerzas espurias.

\section{Diseño metodológico}

El enfoque de esta investigación es de corte post-positivista, con objetivos descriptivos y correlacionales (Costa Vargas, 2005). La fuente de datos proviene de PISA 2012 basada en el uso de una encuesta estandarizada a nivel internacional.

La misma se aplica a una muestra de estudiantes de 15 años de edad al momento de la encuesta, que se encuentran asistiendo a un centro educativo. No se relevan los 
desafiliados. En la edición 2012 fueron relevados 5315 casos, con cobertura y validez nacional para el Uruguay. Por ende la base utilizada se concentra en enseñanza media superior, si bien por cuestiones de rezago también incluye estudiantes que se encuentran en Ciclo Básico. Como señala Cea D'Ancona (2001) los diversos tipos de encuesta suponen costos y beneficios sobre los cuales el investigador debe dar cuenta. En relación a la base PISA enfatizamos que permite generar una información de alcance y validez nacionales y garantizar el acceso a una información con la cual la Administración Nacional de Educación Pública y sus subsistemas se han comprometido. Asimismo, se destacan los altos estándares de exigencia con los cuales PISA elabora y testea sus instrumentos a nivel internacional, lo cual dota de robustez al instrumento. Con las variables reseñadas PISA genera cuatro índices para analizar el clima de los centros educativos: Problemas de clima asociados a estudiantes, Problemas de clima asociados a docentes, Moral docente y Consenso docente. Todos los índices tienen media 0 y desvío de 1, por lo que su rango varía entre -1 y 1.

A efectos de encontrar las variables de la base PISA utilizadas en este estudio se realiza una enumeración de las mismas con comentarios. Las mismas pueden hallarse en el Anexo I. En el presente estudio de desarrollan dos niveles de análisis estadísticos. En el plano descriptivo (primer objetivo), las variables son estudiadas según su distribución, o en su nivel métrico, según las medidas de tendencia central, fundamentalmente su media o promedio. Asimismo, en ocasiones se estudian las diferencias entre los valores promedio de dos sub-poblaciones diferentes. Para saber si dichas diferencias son significativas se ha elegido el análisis Post-hoc según Bonferroni, basado en la distribución T de Student que tiene la ventaja de controlar la tasa de error dividiendo el nivel de significación entre las comparaciones con la tasa de error global (Greenberg, 2006).

Para el segundo objetivo se realiza un análisis correlacional. Tomando en cuenta que todas las variables a relacionar son métricas, se ha elegido el coeficiente $\mathrm{R}$ de Pearson para el estudio de sus relaciones. Por una parte, este coeficiente tiene las ventajas de tener un valor de coeficiente independiente de cualquier unidad utilizada para medir las variables, al tiempo que se robustece con un mayor tamaño de la muestra. Por otra parte, exige que las variables se acerquen a una distribución normal (Field, Miles y Field, 2012). Tomando en cuenta que todas las variables a correlacionar son índices normalizados y validados por PISA, este coeficiente se muestra idóneo para el análisis aquí propuesto.

Para finalizar este apartado se establecen algunas distinciones conceptuales y se realizan varias aclaraciones. En primer lugar, el concepto de escuela refiere a la institucionalidad educativa en un sentido amplio, usualmente vinculada a la idea de centro educativo. En segundo lugar, la implicancia epistemológica de este estudio exige aclarar que todos los resultados aquí expresados refieren a los estudiantes y centros relevados en la cohorte PISA 2012, si bien se omite su sucesiva reiteración para facilitar la lectura del texto. En tercer lugar se señala que todas las variables de PISA se escriben con iniciales mayúsculas para separarlas de los conceptos que representan.

\section{Análisis de resultados}

\section{El Clima educativo}

El Clima escolar está compuesto por una serie de 19 ítems con problemas diversos que pueden encontrarse en los centros. Cada uno de ellos incluye cuatro opciones para indicar la frecuencia de ocurrencia en el centro que van desde nada (1) a mucho (4). De este modo, a mayor número peor valorada es la dimensión del problema. En este plano de análisis cada ítem o problema ha sido respondido con valores entre 1 y 4 según la frecuencia con 
la que el director cree que ocurren en su centro educativo y comprenden: 1 nada, 2 muy poco, 3 en cierta medida y 4 mucho. Por tanto, el valor promedio de las respuestas indica qué tanto se acerca el guarismo a estos valores cualitativos.

Cada uno de ellos está dotado de valor cualitativo en tanto refiere a diferentes elementos que obstruyen el buen clima educativo. Por este motivo resulta importante su estudio individual, de modo de comprender la magnitud con la que ocurre cada fenómeno en la cotidianeidad educativa así como las relaciones entre ellos. Para hacerlo tomamos el promedio numérico de cada ítem, entendiendo que su valor remite a la frecuencia de su ocurrencia. Como vemos en el cuadro 1, según los directores de los centros educativos los problemas que más deben enfrentar en términos de clima escolar refieren, en primer lugar, a tener que enseñar a estudiantes con diferentes niveles de habilidades $(3,01)$, al ausentismo docente (2,87), a los estudiantes que no están siendo motivados como podrían y a los estudiantes que llegan tarde $(2,59)$. Tomando las referencias de respuesta, los directores de centros educativos declararían que estos problemas acontecen en cierta medida. En el otro extremo, los problemas menos declarados, con proximidad a nunca, refieren a la necesidad de enseñar a estudiantes con diferentes orígenes étnicos $(1,31)$, al uso de alcohol o drogas $(1,38)$ y al tener docentes excesivamente severos con los estudiantes $(1,75)$.

Cuadro 1. Promedio de respuesta por ítem de clima educativo

\begin{tabular}{|l|l|}
\hline Variables de clima educativo & Promedio \\
\hline $\begin{array}{l}\text { Profesores que tienen que enseñar a estudiantes con distintos niveles de } \\
\text { habilidad en una misma clase }\end{array}$ & 3,01 \\
\hline Ausentismo docente & 2,87 \\
\hline $\begin{array}{l}\text { Estudiantes que no están siendo motivados para que desarrollen todo } \\
\text { su potencial }\end{array}$ & 2,59 \\
\hline Estudiantes que llegan tarde a las clases & 2,59 \\
\hline Inasistencia de los estudiantes a clases sin autorización & 2,35 \\
\hline Profesores que no atienden las necesidades individuales de los estudiantes & 2,25 \\
\hline Estudiantes que entorpecen el desarrollo de las clases & 2,23 \\
\hline Profesores que llegan tarde a clases & 2,21 \\
\hline Bajas expectativas de los profesores respecto de sus estudiantes & 2,21 \\
\hline Resistencia al cambio por parte del plantel del centro educativo & 2,18 \\
\hline Estudiantes que saltean algunas clases & 2,16 \\
\hline Profesores que no están bien preparados para las clases & 2,09 \\
\hline Estudiantes que faltan el respeto a los docentes & 1,87 \\
\hline $\begin{array}{l}\text { Estudiantes que no asisten a actividades obligatorias (ej. actividades } \\
\text { deportivas) o a salidas didácticas }\end{array}$ & 1,84 \\
\hline Relaciones inadecuadas entre estudiantes y profesores & 1,8 \\
\hline Estudiantes que intimidan o amenazan a otros estudiantes & 1,79 \\
\hline Profesores que son excesivamente severos con los estudiantes & 1,75 \\
\hline Uso de alcohol o drogas ilegales por parte de estudiantes & 1,38 \\
\hline Profesores que tienen que enseñar a alumnos de diversos orígenes étnicos & 1,31 \\
\hline Fuente: Elaboracion propia en base a datos PISA 2012 & \\
\hline
\end{tabular}

Fuente: Elaboración propia en base a datos PISA 2012 
Estos ítems fueron ordenados gráficamente según grado de ocurrencia del fenómeno, omitiendo exclusivamente aquellos cuyas respuestas indicaron que el problema no ocurría nunca. El ordenamiento varía en función del cuadro anterior y puede apreciarse en el Gráfico 1.

Gráfico 1. Problemas de clima educativo (\% de acuerdo desde muy poco, hasta mucho)

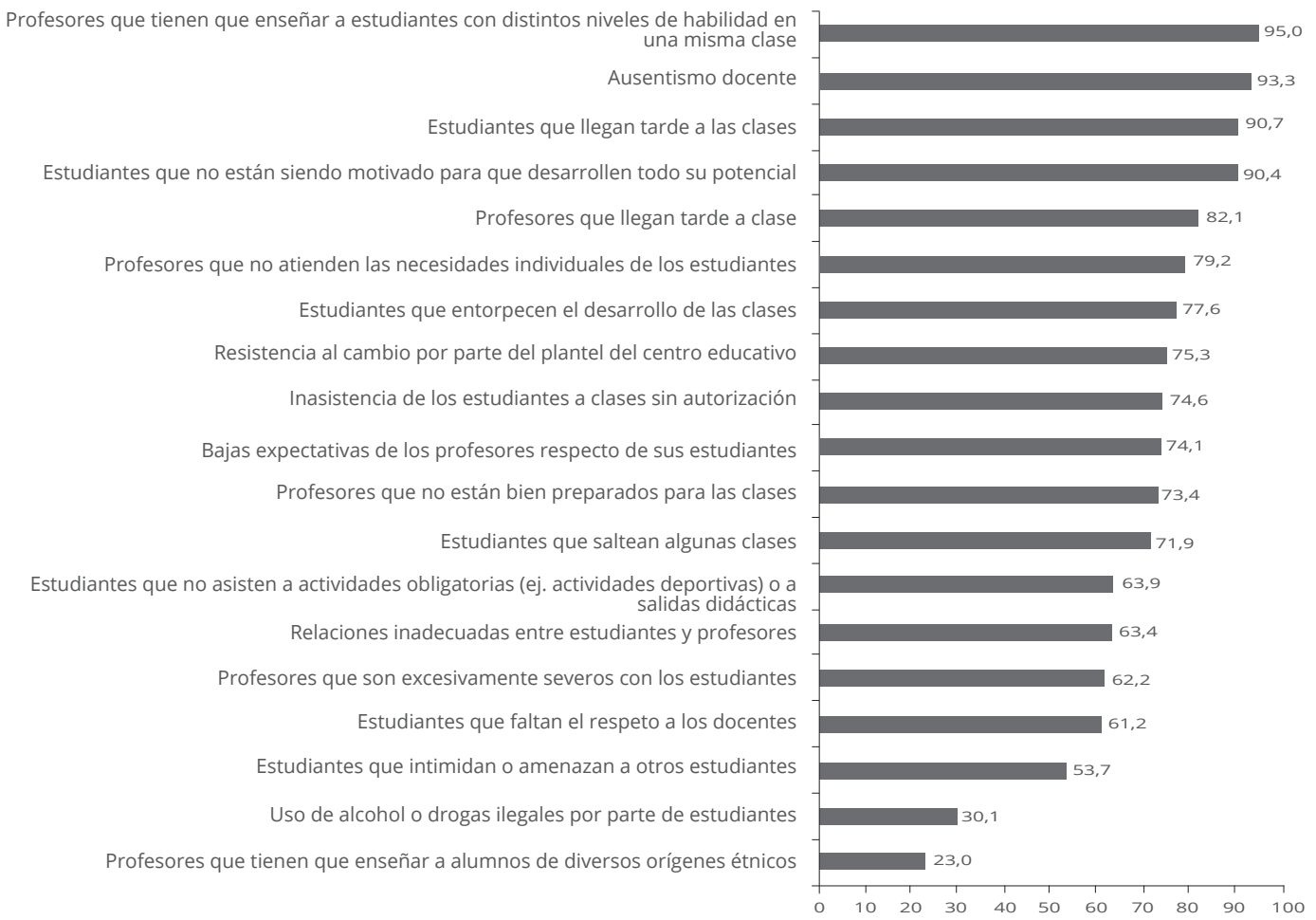

Fuente: Elaboración propia en base a datos PISA 2012

Estos resultados, a diferencia de los presentados en el cuadro 1, marcan el porcentaje de respuestas que señalan que el evento sucedió alguna vez, más allá de su frecuencia. Al respecto, las respuestas parecen coincidir con diagnósticos realizados por diferentes investigadores y especialistas en el tema.

En primer lugar, la dificultad de enseñar a estudiantes con diferentes niveles de habilidad, que es expresada por el $95 \%$ de los directores, se encuentra en los dilemas mismos de la didáctica que sustenta el dispositivo educativo en la estandarización como forma de "enseñar todo a todos, y de la misma manera" (Camilloni, 2007). Asimismo, esto condice con discusiones teóricas y políticas que tienen lugar hoy en el panorama educativo, relativas a la masificación y a la calidad educativa (Tedesco, 2012). En este marco cobra sentido la identificación de la necesidad de que este ítem aparezca como el principal problema enfrentado en los centros educativos, junto con la ubicación en sexto lugar del ítem profesores que no atienden las necesidades individuales de los estudiantes, con un $79,1 \%$ de adhesiones, y la ubicación en séptimo lugar del ítem estudiantes que entorpecen el desarrollo de las clases $(77,5 \%)$ entendido en el marco de las dificultades institucionales enfrentadas para generar propuestas que atiendan a la individualidad y ordenen el flujo de estudiantes dentro y fuera de las aulas (Rivero, 2013). Del mismo modo, estos elementos indican la relevancia de la dimensión cultural del clima, señalada por Fernández (2004). 
En segundo lugar aparece como problema importante el ausentismo docente, con un 93,2 $\%$ de directores que mencionan su ocurrencia en algún nivel. A esto podríamos agregar la problemática de los docentes que Ilegan tarde a clase (82,1\%), la resistencia al cambio por parte del plantel docente $(75,6 \%)$ y los profesores que no están bien preparados para las clases $(73,4 \%)$. Estos elementos se encuentran presentes en la dimensión grupal-motivacional de Fernández (2004), y puede leerse en función de la literatura reciente que indica una serie de problemas actuales existentes en torno a la profesión docente (INEEd, 2014). Los mismos señalan la insuficiencia en la formación, marcada por una baja tasa de finalización de los estudios, la dedicación dividida en diferentes centros y el no reconocimiento salarial, tanto en relación con otros profesionales como en relación al trabajo extra-aula, lo que se encuentra implícito en la tarea educativa.

En tercer lugar surge con relevancia el hecho de que los estudiantes llegan tarde a clase $(90,7 \%)$, falten sin autorización (74,6\%), salteen clases $(71,9 \%)$ y no concurran a actividades obligatorias o a salidas didácticas (64\%). Por un lado, estos elementos han sido estudiados y pueden vincularse a una cultura de la impuntualidad que ubica a Uruguay como el país que registra el mayor guarismo de llegadas tarde (OECD, 2013). Por otro, alertan sobre la posibilidad de generar escolaridades de baja intensidad que desgasten el lazo entre los estudiantes y la institución educativa (Kessler, 2004; Mancebo y Méndez, 2012).

En cuarto lugar, se identifica con relevancia que el $90 \%$ de los directores declaren que en sus centros haya estudiantes que no están siendo motivados para que desarrollen todo su potencial, en tanto que un $74 \%$ de ellos entienden tener docentes con bajas expectativas respecto a sus estudiantes. Esta problemática ha sido reseñada por Tedesco (2012) en torno al efecto Pigmalión, según el cual la expectativa de éxito de los docentes es una clave fundamental para los resultados educativos que se obtengan.

En último lugar, si ítems como las relaciones inadecuadas entre docentes y estudiantes, profesores que son muy severos con sus estudiantes, estudiantes que faltan el respeto a los docentes y estudiantes que intimidan o amenazan a otros estudiantes no se encuentran como los problemas más expresados por los directores, estos superan, en todos los casos, a la mitad de los centros estudiados. Los elementos antedichos entran en diálogo con los estudios acerca de la violencia en el espacio educativo (Kaplan, 2012; Viscardi y Alonso, 2013), que entienden al centro educativo como un espacio de conflictos, por lo que deben generar políticas activas para promover la convivencia.

\section{La Moral docente}

La variable Moral docente en PISA está compuesta por cuatro ítems que comprenden afirmaciones con las que los directores deben indicar su grado de conformidad. El valor 1 es muy de acuerdo, el 2 es de acuerdo, el 3 en desacuerdo y el 4 muy en desacuerdo. Para analizarlos desagregamos sus respuestas y generamos un resumen con valores entre 1 y 4. En este caso, dada la codificación de PISA, un mayor promedio indica mayores problemas, es decir, un peor estado del ítem moral docente.

Si observamos el porcentaje de acuerdo con los diferentes ítems constatamos que los directores expresan altos niveles de aprobación, de entre el 87,8\% y el 93,7\%, centrándose el mayor valor en el plano académico y el menor en el entusiasmo (Gráfico 2). 
Gráfico 2. Moral docente (\% de respuestas de acuerdo y muy de acuerdo con el ítem)

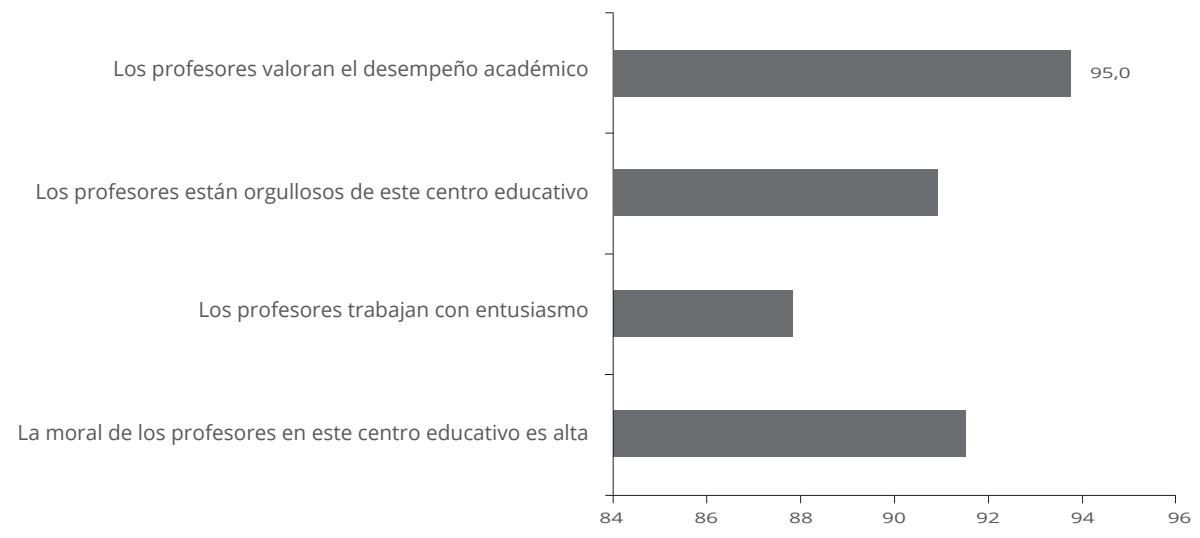

Fuente: Elaboración propia en base a datos PISA 2012

Si bien esto expresa un panorama altamente positivo, los datos se encuentran en contraste con los resultados del Censo Nacional Docente según el cual menos de la mitad de los docentes de todos los subsistemas se encontraban satisfechos con el clima de trabajo en los centros, y menos de la cuarta parte en términos del local y los recursos didácticos del centro (Administración Nacional de Enseñanza Pública - Consejo Directivo Central -ANEP-CODICEN-, 2008). En este sentido, es probable que exista una distancia entre estos diagnósticos y los de PISA, que toman a los directores como fuente de información, lo que constituye algo sobre lo que profundizar.

\section{El Consenso Docente}

De modo similar que con el constructo anterior PISA releva problemas en torno al consenso docente, de modo que los valores cercanos a 1 indican un mayor grado de acuerdo y los valores cercanos a 4 un menor acuerdo con el ítem. Como podemos apreciar en el Gráfico 3 , el mayor grado de acuerdo se encuentra en el interés en utilizar nuevos métodos y prácticas de enseñanza, mientras que el menor acuerdo se encuentra en la adaptación de estándares académicos a los niveles y necesidades de los estudiantes.

Gráfico 3. Consenso docente (\% de acuerdo con cada ítem)

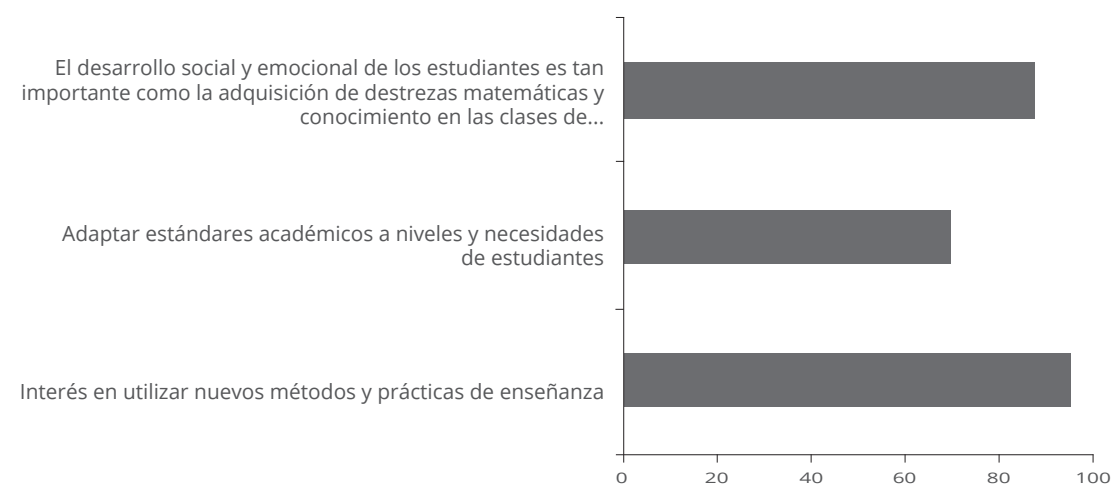

Fuente: Elaboración propia en base a datos PISA 2012 
Esta diferencia cobra sentido si consideramos que los directores mencionaron la necesidad de enseñar a estudiantes con diferentes niveles como el principal problema del clima educativo. Parecería, entonces, que esta es una problemática identificada desde los directores relevados en PISA 2012, y sobre la cual se visibiliza un menor consenso por parte de los equipos docentes acerca de cómo trabajar en ella.

Se destaca, entonces, que la heterogeneidad de niveles y rendimientos es identificada como un problema importante por parte de los directores de los centros educativos a nivel nacional. Ello condice con los diagnósticos que expresan que la adaptación a los estándares académicos (conocida bajo el rótulo de adecuación pedagógica) resulta una problemática central que pone en jaque a las prácticas y formatos escolares en términos de integrar o estigmatizar a los sujetos pedagógicos (Bordoli, 2012).

En contrapartida, el consenso en el interés en utilizar nuevos métodos y prácticas de enseñanza cobra sentido si, como señala el Censo Nacional Docente (ANEP-CODICEN 2008), la mayor satisfacción docente proviene del ejercicio de la docencia directa. En contrapartida, este deseo innovador expresado en el ítem contrasta con el bajo porcentaje de docentes titulados, fundamentalmente en la enseñanza media (INEEd, 2014).

\section{El clima educativo como constructo}

Como fue señalado, PISA elabora sus constructos y variables a partir de una teoría general pero corroborar que las mismas se relacionen entre sí parte de una constatación empírica en cada país.

En tal sentido, es de interés conocer si los componentes antes reseñados se relacionan entre sí como un gran constructo de clima educativo o si se trata de variables sin interrelación fáctica.

Para estudiar el clima educativo a partir de sus factores se tomarán los índices de constructo generados por PISA. Los mismos tienen la ventaja de estandarizarse según las mismas pautas en todos los países. El índice tiene la desventaja de desanclarse de los ítems concretos que forman la variable latente pero gana en la robustez de su comparabilidad (OECD, 2014).

Estos índices tienen una media 0 y una varianza de 1, por lo que varían entre -1 y 1 . La escala está estructurada de modo que valores positivos representan mayores niveles de acuerdo con el ítem, y valores negativos menores niveles de acuerdo.

Para estudiar que las variables efectivamente den cuenta de un gran constructo sobre clima educativo, fueron correlacionados los cuatro índices (Cuadro 2). 
Cuadro 2. Correlación de variables de clima educativo

\begin{tabular}{|c|c|c|c|c|c|}
\hline & & $\begin{array}{l}\text { Factores de } \\
\text { clima } \\
\text { asociados } \\
\text { a docentes }\end{array}$ & $\begin{array}{l}\text { Factores } \\
\text { de clima } \\
\text { asociados a } \\
\text { estudiantes }\end{array}$ & $\begin{array}{l}\text { Moral } \\
\text { docente }\end{array}$ & $\begin{array}{l}\text { Consenso } \\
\text { docente }\end{array}$ \\
\hline $\begin{array}{l}\text { Factores de clima } \\
\text { asociados a } \\
\text { docentes }\end{array}$ & $\begin{array}{l}\text { Correlación } \\
\text { de Pearson } \\
\text { Sig. (bilateral) } \\
\text { N }\end{array}$ & $\begin{array}{l}1 \\
5313+2 \\
\end{array}$ & $\begin{array}{l}, 638^{* *} \\
0,000 \\
5313\end{array}$ & $\begin{array}{l}, 314 * * \\
.000 \\
5313 \\
\end{array}$ & $\begin{array}{l}, 152 * \star \\
, 000 \\
5313\end{array}$ \\
\hline $\begin{array}{l}\text { Factores de clima } \\
\text { asociados a } \\
\text { estudiantes }\end{array}$ & $\begin{array}{l}\text { Correlación } \\
\text { de Pearson } \\
\text { Sig. (bilateral) } \\
\text { N }\end{array}$ & $\begin{array}{l}, 638 * * \\
0,000 \\
5313\end{array}$ & $\begin{array}{l}1 \\
5313\end{array}$ & $\begin{array}{l}, 585^{* *} \\
0,000 \\
5313\end{array}$ & $\begin{array}{l}, 358 * * \\
, 000 \\
5313\end{array}$ \\
\hline Moral docente & $\begin{array}{l}\text { Correlación } \\
\text { de Pearson } \\
\text { Sig. (bilateral) } \\
\text { N }\end{array}$ & $\begin{array}{l}, 314 * * \\
, 000 \\
5313\end{array}$ & $\begin{array}{l}, 585 * * \\
0,000 \\
5313\end{array}$ & 5315 & $\begin{array}{l}.419 * * \\
, 000 \\
5315\end{array}$ \\
\hline Consenso docente & $\begin{array}{l}\text { Correlación } \\
\text { de Pearson } \\
\text { Sig. (bilateral) } \\
\text { N }\end{array}$ & $\begin{array}{l}, 152 * * \\
.000 \\
5313\end{array}$ & $\begin{array}{l}, 358 * * \\
, 000 \\
5313\end{array}$ & $\begin{array}{l}.419 * * \\
.000 \\
5315\end{array}$ & 5315 \\
\hline
\end{tabular}

Fuente: Elaboración propia en base a datos PISA 2012

El primer paso para el análisis de estas relaciones supone estudiar si los factores de clima docente se vinculan con los factores de clima estudiantil. Al respecto, es visible que existe una asociación positiva, intensa y fuerte entre ambas variables $(r=0,638)$, lo que reviste sentido desde una perspectiva ecológica: las problemáticas de clima vinculadas con los docentes se relacionan con aquellas centradas en los estudiantes.

Asimismo, ambas se encuentran positivamente relacionadas con la variable moral docente, especialmente la que refiere a los elementos de clima vinculados a los docentes $(r=0,585)$, pero también en los centrados en los estudiantes ( $r=0,314)$.

Finalmente, cuando estudiamos el vínculo con el consenso docente encontramos que presenta una correlación significativa leve con los factores de clima vinculados a estudiantes $(r=0.152)$, pero muestra mejores relaciones con respecto a los factores de clima vinculados a docentes $(r=0.358)$ y mayor aún con la moral docente $(r=0.419)$

En otras palabras, en relación al cuestionario PISA 2012 respondido por los directores se entiende que, en aquellos centros donde hay un mejor clima educativo existen Moral y Consenso docentes mayores. Estas variables conforman un cuadrilátero que se presenta con relativa fortaleza dadas las altas correlaciones que presentan entre sí, y por tanto es posible suscribir al planteo del clima educativo en un sentido amplio. 
Al mismo tiempo cabe notar ciertas especificidades que parecen delinearse en tal relación. Por un lado, la fortaleza del vínculo entre los factores docentes y estudiantiles en el clima. Por otro, las altas relaciones entre la moral docente, tanto con los factores docentes de clima como con el consenso docente.

En términos generales es posible afirmar que, desde la perspectiva de los directores, en aquellos centros donde hay mejor trato entre docentes y estudiantes, mayores expectativas, mejor clima de diálogo y cercanía hay también mayores expectativas docentes de los resultados y voluntad de desarrollo conjunto.

Retomando los enfoques teóricos esbozados, el clima educativo parece visualizarse fácticamente como una gran categoría de sentido que rige el habitar y el entendimiento en la institución escolar. Esto abona una línea de pensamiento institucionalista que coloca al centro educativo como una unidad de sentido propio, con reglas de funcionamiento específicas sobre las cuales es posible trabajar para mejorar la experiencia educativa de quienes conviven en su seno. Al mismo tiempo, esto se muestra relevante en términos de lograr el desarrollo de los objetivos educativos emanados de la institucionalidad misma.

\section{Conclusiones y reflexiones finales}

En este trabajo se ha analizado el clima educativo de los centros PISA 2012, concebido en múltiples dimensiones. Por un lado, se estudió qué problemáticas predominan y cómo se distribuyen según algunas variables de interés. Por otro lado se buscó vincularlo a problemáticas que en la literatura aparecen como relevantes.

La perspectiva adoptada en este trabajo nos permite observar la relación entre diferentes fenómenos que tienen lugar en la cotidianeidad educativa de los centros, los que son condensados en tanto variables. Esto tiene la ventaja de lograr establecer las diferentes partes involucradas y analizarlas de forma separada. Enfrenta el desafío, sin embargo, de tener en cuenta que las mismas acontecen de forma ecológica y que son vividas por los actores desde un punto de vista integrado. Por esto, comprender el sentido de lo que ocurre implica siempre realizar una mirada de los resultados a partir de la teoría, integrando los datos con la perspectiva de los actores, lo que se busca realizar en este apartado. En base a ello se realizan cuatro reflexiones finales.

En primer lugar, sobre los resultados descriptivos, el análisis realizado permite ordenar diferentes diagnósticos presentes en la literatura actual sobre los problemas educativos. Los dilemas en torno a los múltiples niveles de los estudiantes se encuentran en el seno de un dispositivo escolar estandarizador; los problemas en el plantel docente se ubican en la base de los desafíos del centro, y la capacidad de cautivar a los estudiantes para seguir las normas escolares habla de las dificultades de legitimar el sistema educativo en un espacio sociocultural específico. Estos problemas han sido ampliamente desarrollados teóricamente, y los resultados aquí presentados colaboran en el conocimiento de la importancia de dichos problemas en la mirada de los directores de los centros de enseñanza media.

En segundo lugar se señala el surgimiento de algunos datos de particular interés, como es que en relación al Consenso Docente el hecho de que el elemento de menor acuerdo refiera a la adaptación de la propuesta y los estándares educativos a la diversidad de habilidades de los estudiantes. En relación a la Moral Docente, que el alto grado de acuerdo general contrasta fuertemente con los diagnósticos del malestar docente (Esteve, 1987), y se abona así idea sobre el clima que parece alejarse de los climas tóxicos (Milicic y Arón, 2014). 
Al respecto, esta diferencia en los resultados puede tener una raíz metodológica pues al realizarse un relevamiento por cuestionario, el clima es entendido como sumatoria de ítems concretos, desagregados a fenómenos específicos de la cotidianeidad educativa. En contraposición, es posible que los abordajes cualitativos releven insumos discursivos que por su lógica de producción llevan a lugares comunes del entendimiento, donde la noción de malestar se encuentra fuertemente asentada.

Otro elemento a considerar resulta del hecho de que la información provenga de los directores de los centros, lo que permite cierto sesgo propio de una unidad de registro situada desde el poder. En cualquier caso, las distancias entre estos datos y los antecedentes suponen una cuestión interesante a profundizar.

En tercer lugar, tomando en cuenta todos los elementos reseñados, en función del concepto de clima escolar conceptualizado por Fernández (2004), resultan dos elementos a resaltar. Primero, la dimensión cultural aparece de forma particularmente problemática según lo aquí analizado. Las expectativas de logro se ven interferidas por problemas tales como la heterogeneidad en los niveles y la falta de motivación a estudiantes. En este sentido, en términos de la visión de la escuela esto genera dificultades en la adopción de los estándares académicos a las necesidades estudiantiles (como expresan los resultados de la variable Consenso Docente), y en el desarrollo del personal docente de acuerdo con objetivos de centro (como indica la variable Comunicación de Objetivos). Segundo, en lo que refiere a la dimensión grupal- motivacional encontramos un escenario diverso que, en la visión de los directores, parece positivo. Por una parte parece existir cooperación en la resolución de problemas cotidianos en el aula, como lo indican los altos valores de la variable Desarrollo Docente. Por otra parte, los altos valores del constructo Moral Docente parecen establecer un escenario propicio para la afiliación grupal.

Por último, parece importante señalar que la constatación de que todos los constructos se relacionan entre sí pone de relieve que el clima educativo es una categoría estructural que opera en los centros educativos, y por lo tanto, que tiene valor para comprender cómo estos centros funcionan. En tal sentido se marcan dos desafíos a futuro: el primero de ellos es conocer las formas en que las personas que habitan el espacio escolar significan la noción de clima, buscando armonizar las miradas de los datos y de los sujetos. Avanzar en la realización de estudios cualitativos puede aportar a tales objetivos. El segundo es analizar el clima de forma causal, buscando conocer el impacto que pueda tener en el mejoramiento de otras variables educativas de interés. Esto sería una colaboración sustantiva tanto a nivel del sistema educativo en términos globales como de la dirección y planificación de los centros.

\section{Referencias bibliográficas}

ANEP-CODICEN (2008). Censo Nacional Docente. Disponible en: https://www.mec.gub.uy/ innovaportal/file/91321/1/1er-censo-nacional-2007.pdf

Blanco, E. (2009). Eficacia escolar y clima organizacional: apuntes para una investigación de procesos escolares. Estudios Sociológicos 2009, XXVII (mayo-agosto). Disponible en: http:// www.redalyc.org/articulo.oa?id=59820676011

Bordoli, E. (2012). Nuevos formatos escolares: entre la integración y la estigmatización de los sujetos pedagógicos. En Vulnerabilidad y exclusión. Aportes para las políticas sociales (pp. 71-83). Montevideo: MIDES 
Bourdieu, P. y Passeron, J.C. (1995). La reproducción. Elementos para una teoría del sistema de la enseñanza. México D.F.: Fontamara.

Camilloni, A. et al. (2007). Didáctica general y didácticas específicas. En El saber didáctico. Buenos Aires: Paidós

Cea D'Ancona, M.A. (2001). La investigación social mediante encuesta. En Metodología cuantitativa: estrategias y técnicas de investigación social (pp. 239-291). Madrid: Síntesis.

Charlot, B. (2008). La relación con el saber. Montevideo: Trilce.

Coleman, J., Hoffer, T. y Kilgore, S. (1982). High School achievements: Public, Catholic and Private School compared. New York: Basic Books.

Costa Vargas, A. (2005). Guía para elaborar una propuesta de investigación. En Revista Educación, 29(2). pp. 77-97.

Dubet, F. (2006). El declive de la institución: profesores, sujetos e individuos en la modernidad. Barcelona: Gedisa Editorial

Durlak, J., Weissberg, R., Dymnicki, A., Taylor R. \& Schellinger, K. (2011). The impact of enhancing students' social and emotional learning: a meta-analysis of school-based universal interventions. En Child Development, 82(1) pp. 405-432.

Duschatzky, S. (2013). Veo, veo... ¿Qué ves? Percepciones más allá (o más acá) de la violencia. Disponible en: https://dialnet.unirioja.es/descarga/articulo/4968419.pdf

Esteve, J.M. (1987). El malestar docente, Barcelona: Laia.

Esteve, J.M. (2006). Identidad y desafíos de la condición docente. En: Tenti Fanfani, E. (Comp.) El oficio del docente. Vocación, trabajo y profesión en el siglo XXI. (pp. 19-69). Buenos Aires: Siglo XXI/lipe-Unesco/Fundación Osde

Farrington, C.A., Roderick, M., Allensworth, E., Nagaoka, J., Keyes, T.S., Johnson, D.W. \& Beechum, N.O. (2012). Teaching adolescents to become learners. The role of noncognitive factors in shaping school performance: A critical literature review. Chicago: University of Chicago. Consortium on Chicago School Research.

Disponible en: https://consortium.uchicago.edu/sites/default/files/2018-10/ Noncognitive\%20Report_0.pdf

Fernández, T. (1996). Análisis organizacional de secundaria del ciclo básico del área metropolitana: teoría, métodos y hallazgos. Revista de Facultad de Ciencias Sociales. FCS - DS Año 11 (Número 12), pp. 132-143

Fernández, T. (1999). Análisis organizacional en educación: un estudio muestral sobre gerencia, iniciativas de cambio, clima y eficacia en liceos públicos del Uruguay. Documento de trabajo N. ${ }^{\circ} 46$ UR. FCS-DS. 
Fernández, T. (2004). Clima organizacional en las escuelas: un enfoque comparativo para México y Uruguay. REICE - Revista Electrónica Iberoamericana sobre calidad, eficacia y cambio en educación 2004, Vol. 2(2). Disponible en: https://revistas.uam.es/index.php/reice/article/ view/5548

Fernández, T. (2010). Factores escolares y desafiliación en la enseñanza media superior de Uruguay (2003-2007). Montevideo: UdelaR

Fernández, T. y Ríos, A. (2014). El tránsito entre ciclos en la educación media y superior de Uruguay. Montevideo: U.R.. CSIC.

Field, A., Miles, J. \& Field, Z. (2012). Discovering statistics using R. London: SAGE.

Filardo, V. y Mancebo, M.E. (2013). Universalizar la educación media en Uruguay: ausencias, tensiones y desafíos. Montevideo: U.R. FCS. CSIC.

Filgueira et al. (2014). La educación prioridad país: aportes a la construcción de una educación genuinamente inclusiva. Consultado el 21/03/2014. Disponible en: https://issuu.com/ fundacionceibal/docs/educacion_final_vilaro

Frigerio, G., Poggi, M. y Tiramonti, G. (1992). Las instituciones educativas. Cara y ceca. Buenos Aires: Troquel.

Fullan, M. y Hargreaves, A. (1999). La escuela que queremos. Buenos Aires: Amorrortu

Greenberg, B. (2006). The basic practice of statistics. New York: W.H. Freeman and Company.

INEEd (Instituto Nacional de Evaluación Educativa) (2014). Informe sobre el estado de la educación en Uruguay 2014. Montevideo: INEEd.

INEEd (Instituto Nacional de Evaluación Educativa) (2015). Habilidades no-cognitivas y desempeños en matemática entre los estudiantes uruguayos evaluados en PISA 2012. Montevideo: INEEd.

Kaplan, C. (2009). Violencia escolar bajo sospecha. Buenos Aires: Editorial Miño y Dávila.

Kaplan, C. (2012) (Coord.). Violencias en plural. Sociología de las violencias en la escuela. Buenos Aires: Editorial Miño y Dávila.

Kessler, G. (2004). Sociología del delito amateur. Buenos Aires: Paidós.

Mancebo, M.E. y Méndez, N. (2012). La exclusión educativa en los países del Cono Sur. Aproximación conceptual y dimensionamiento. En Revista de Ciencias Sociales N. ${ }^{\circ} 30$. pp. 117-138.

Martinis, P. y Falkin, C. (2017). Aspectos pedagógicos y de política educativa involucrados en los procesos de universalización del derecho a la educación. (Artículo inédito, en el marco de Proyectos de Artículo 2. UdelaR) Montevideo: FHUCE 
McDermott, R.P. (2001). La adquisición de un niño por una discapacidad de aprendizaje. En Chaiklin, S. y Lave, J. Estudiar las prácticas. Capítulo 10. (pp. 291-330). Buenos Aires:Amorrortu.

Milicic, N. y Arón, A. (2014). Climas sociales tóxicos y climas sociales nutritivos para el desarrollo personal en el contexto escolar. Disponible en: http://www.buentrato.cl/pdf/est_inv/conviv/ ce aron2.pdf

OECD (Organization for Economic Cooperation and Development) (2013). PISA 2012 Results: ready to learn: students' engagement, drive and self-beliefs (Volume III), PISA, OECD Publishing.

OECD (Organization for Economic Cooperation and Development) (2014). Disponible en: https://www.oecd.org/pisa/pisaproducts/PISA \%202012 \%20Technical \%20Report_Chapter \%2016.pdf

Ravela, P. et al. (1999). Factores institucionales y pedagógicos explicativos de los aprendizajes. Cuarto informe de la evaluación nacional de aprendizajes de los sextos años de educación primaria. Montevideo: UMRE - Mecaep - ANEP

Rivero, L. (2013). Proyecto pedagógico, legitimidad y control. Exploración de la violencia en dos liceos montevideanos. Tesis de grado de la Licenciatura en Sociología. Montevideo: FCS - UdelaR.

Rivero, L. (2015). Trayectorias educativas tras el concepto ni-ni. En Cuadernos de CCSS y PPSS. Tomo I, pp. 141-160. Montevideo: MIDES-UR.

Rossi Silva, J. (2001). El malestar docente: hacia la reconstrucción de la fe perdida. Montevideo: Doble Click.

Tedesco, J.C. (2000). Educar en la sociedad del conocimiento. Buenos Aires: Fondo de Cultura Económica.

Tedesco, J.C. (2012). Educación y justicia social en América Latina. Buenos Aires: Fondo de Cultura Económica.

Viscardi, N. y Alonso, N. (2013). Gramáticas de la convivencia. Montevideo: ANEP.

Viscardi, N. y Alonso, N. (2015). Convivencia, participación y formación de ciudadanía. Un análisis de sus soportes institucionales en la educación pública uruguaya. Montevideo: ANEP, Mosca.

Viscardi, N. y Habiaga, V. (2017). El derecho a la educación en disputa: dinámicas de resistencia y dinámicas de exclusión. (Artículo inédito, en el marco de Proyectos de Artículo 2. UdelaR) Montevideo: FHUCE. 


\section{Anexos}

\section{Anexo I. Variables utilizadas}

\begin{tabular}{|c|c|c|}
\hline Constructo & Variable & $\begin{array}{l}\text { Nombre en la base } \\
\text { de datos PISA } 2012\end{array}$ \\
\hline Tamaño del centro & $\begin{array}{l}\text { Índice de tamaño de } \\
\text { centro }\end{array}$ & SCHSIZE \\
\hline \multirow{4}{*}{ Clima educativo } & Clima educativo & $\begin{array}{ll}\text { SC22Q01 SC22Q02 } \\
\text { SC22Q03 SC22Q04 } \\
\text { SC22Q05 SC22Q06 } \\
\text { SC22Q07 SC22Q08 } \\
\text { SC22Q09 SC22Q10 } \\
\text { SC22Q11 SC22Q12 }\end{array}$ \\
\hline & & $\begin{array}{l}\text { SC22Q13 SC22Q14 } \\
\text { SC22Q15 SC22Q16 } \\
\text { SC22Q17 SC22Q18 } \\
\text { SC22Q19 }\end{array}$ \\
\hline & $\begin{array}{l}\text { Índice de problemas } \\
\text { de clima asociado a } \\
\text { docentes }\end{array}$ & TEACCLIM \\
\hline & $\begin{array}{l}\text { Índice de problemas } \\
\text { de clima asociado a } \\
\text { estudiantes }\end{array}$ & STUDCLIM \\
\hline \multirow[t]{2}{*}{ Moral docente } & Moral docente & $\begin{array}{l}\text { SC26Q01 SC26Q02 } \\
\text { SC26Q03 SC26Q04 }\end{array}$ \\
\hline & $\begin{array}{l}\text { Índice de moral } \\
\text { docente }\end{array}$ & TCMORALE \\
\hline \multirow[t]{2}{*}{ Consenso docente } & Consenso docente & $\begin{array}{l}\text { SC27Q01 SC28Q02 } \\
\text { SC29Q01 }\end{array}$ \\
\hline & $\begin{array}{l}\text { Índice de consenso } \\
\text { docente }\end{array}$ & TCFOCST \\
\hline
\end{tabular}

\section{Notas}

*Facultad de Ciencias Sociales, Universidad de la República (Uruguay).

${ }^{1}$ Doctor (Cand.) en Sociología y Diploma en Juventud y Políticas Públicas, Universidad de la República (Uruguay). Magister en Educación, Universidad ORT Uruguay. Licenciado en Sociología, Universidad de la República (Uruguay). Docente de Metodología de la investigación y Coordinador adjunto de la Licenciatura en Sociología, Universidad de la República (Uruguay). Investigador en educación y trabajo, con énfasis en la cultura del trabajo docente, y en la convivencia escolar. 